Grundlagen 


\title{
Entwicklungslinien einer interdisziplinären Begriffsgeschichte von Grenze
}

\author{
Falko Schmieder
}

\begin{abstract}
Der Beitrag rekonstruiert Entwicklungslinien und Schauplätze des Grenzbegriffs. Neben der politisch-territorialen Bedeutung von Grenze im Zusammenhang der Entstehung des modernen Staates interessieren vor allem die Übertragungen des Grenzbegriffs auf andere Wissensfelder, von der Mathematik und Philosophie über die Ökonomie bis hin zur Ökologie. Als übergreifende Merkmale werden u.a. die Temporalisierung, Politisierung und Verflüssigung des Grenzbegriffs herausgearbeitet. Die Spannungen und Widersprüche der verschiedenen Grenzvokabulare verweisen auf ungelöste politisch-soziale Probleme, die zugleich die Begriffsentwicklungen vorantreiben.
\end{abstract}

\section{Schlagwörter}

Entgrenzung, Grenzüberschreitung, Globalisierung, Geopolitik, Ökologie

\section{Zur Einführung1}

Ein Charakteristikum vieler neuerer Auseinandersetzungen mit dem Thema Grenze ist das interdisziplinäre Forschungsinteresse, das auch die folgende Begriffsgeschichte motiviert. Es ergibt sich vor allem aus der Erfahrung, dass durch die Prozesse der Globalisierung traditionelle Grenzregime und Grenzvorstellungen unter beschleunigten Veränderungsdruck geraten. Bislang disziplinär oder sektoral ausdifferenzierte und voneinander weitgehend unabhängige Grenzbegriffe vermischen sich und geraten in Konflikt miteinander. Darüber hinaus entstehen neue Problem- und Anwendungsfelder des Grenzbegriffs (wie etwa auf dem Feld der politischen Ökologie), die mit überkommenen Bestimmungen ins Verhältnis zu setzen sind. Die vorliegende Darstellung muss sich aus methodischen wie auch aus Platzgründen weitgehend auf die deutsche Sprachentwicklung und im Wesentlichen darauf beschränken, längerfristige Entwicklungstrends sowie wichtige Knotenpunkte und Wandlungen im Verständnis des Begriffswortes und mit ihm zeittypisch verknüpfter Begriffe und Begriffsfelder zu skizzieren. Verbindungen zur Sozialgeschichte sowie vor allem auch die Einbindungen der Begriffe in Praktiken und institutionelle Arrangements können bestenfalls angedeutet werden.

\section{Grenze, politisch-territorial: vorneuzeitliche Entwicklungen}

Im Vergleich mit anderen europäischen Sprachen weist die deutsche Sprachentwicklung des Begriffs Grenze mindestens zwei Besonderheiten auf: zum einen existiert in anderen Sprachen eine Vielzahl von Ausdrücken zur Bezeichnung jeweils spezifischer Bedeutungsfelder, die im Deutschen oft unter dem einen Terminus Grenze gefasst werden, zum anderen ist die moderne Grenzterminologie in den anderen Sprachen eng mit der altokzidentalen Tradition verknüpft, während der in der deutschen Sprache dominierende Grenzbegriff aus dem mittelalterlichen

1 Der vorliegende Beitrag ist in Zusammenarbeit mit dem vom spanischen Wirtschafts- und Wissenschaftsministerium geförderten Forschungsprojekt FFI2017-82195-P entstanden. 
Slawischen, genauer dem Pomoranischen entlehnt ist. Anders als die moderne Entwicklung der Wortbedeutung von Grenze, für die materialreiche und vor allem interdisziplinär verfahrende Studien weitgehend fehlen (vgl. Medick 1995, S. 216; Wokart 1995, S. 278; Böckler 2003), ist die frühe Wortgeschichte relativ gut erschlossen (vgl. Kolb 1989; Schmale 1998). Sie setzt mit dem Terminus granicalgraniz ein, der Trennungslinien zwischen privaten Grundstücken und politisch-territorialen Gebilden bezeichnete und erstmals im Laufe des 12. Jahrhunderts als Fremdwort und im 13. Jahrhundert als Lehnwort im Zusammenhang der Regelung von Grenzstreitigkeiten und Landteilungen zwischen dem Deutschen Orden und den slawischen Fürsten verwendet wurde. Der Ausdruck granica hatte im Altslawischen ursprünglich die Eiche bedeutet (vgl. Hoke 1971, S. 1802). Als Folge der Verwendung des Baumes als Grenzzeichen hatte das Wort dann die Bedeutung des Grenzbaumes und schließlich allgemein der Grenze (Grenzlinie) angenommen. Er trat in dieser Bedeutung zunächst neben das deutsche Wort Marck bzw. Landmarken, das Grenzen vor allem als räumlich ausgedehnte Bereiche zwischen unterschiedlichen Herrschaftsgebieten erfasste und noch nicht deutlich zwischen Grenze im Sinne von Grenzlinie und den an bzw. auf ihr liegenden Gebieten unterschied (vgl. Böckler 2003, S. 179).

Wie sich anhand von Lehns- und Besitzurkunden sowie von Städtechroniken verfolgen lässt, dringt der Ausdruck im 14. und 15. Jahrhundert in regional und zeitlich variierenden Schreibweisen (u.a. granizze, gränze, grenitze, grenytze, grentz, grenicz, grenice, grenitzte) nach Süden und Westen vor. Laut Deutschem Wörterbuch hatte Martin Luther „geradezu eine Vorliebe für das Wort“ (Hübner 1919/1935, Sp. 125). Seine Bibelübersetzung trug maßgeblich dazu bei, dass es „namentlich seit den dreißiger Jahren des 16. Jahrhunderts auf hd. Boden allgemeine Verbreitung findet, und zwar zuerst in den Gebieten, die sich der Reformation öffneten“ (ebd.). Wie ein Vergleich mit anderen Bibelübersetzungen und zeitgenössischen Schriften zeigt, war der Sprachgebrauch aber noch vielfältig und eine Fülle sinnverwandter Ausdrücke unterschiedlicher Herkunft im Gebrauch, wie landmarcken, marck steyn, marck, aber auch anstösz, frontier, anrein, scheyde u.a.m.

Wenn sich Grenze allmählich immer mehr durchsetzte und das ältere Marck zurück- und schließlich fast ganz verdrängte, ${ }^{2}$ so liegt das auch daran, dass der neue Ausdruck schärfer die Grenze als Trennungslinie fokussierte. Der lange und keineswegs linear verlaufende Durchsetzungsprozess wurde später im Anschluss an eine Arbeit von Helmholt (1896) unter der Formel „vom Grenzsaum zur Grenzlinie“ zu einem Paradigma der historischen Grenzforschung. ${ }^{3}$ Seine sozialhistorische Voraussetzung ist der Übergang von der mittelalterlichen zur neuzeitlichen Gesellschaft, genauer die Herausbildung neuzeitlicher Formen der Staatlichkeit und der Durchsetzung territorialstaatlicher Herrschaftsstrukturen. Der damit verbundene Prozess der Rationalisierung (Linearisierung, Verrechtlichung) der Grenze reflektiert sich auch in anderen Sprachen. Eine Parallele zur Ablösung der Grenze von Marck bildet im Französischen die Ablösung von limes/fines durch frontiere (vgl. Febvre 1928/1988). Frontiere hat eine militärische Bedeutung, die auf den Staat als grenzziehende Instanz verweist. Lucien Febvre führt die Bedeutungsveränderungen der Grenzbegrifflichkeit auf Personengruppen zurück, die im Dienste des entstehenden territorialen Staates standen, unter ihnen vor allem Militärs, Geo-

2 Der ältere Sprachgebrauch klingt in Bezeichnungen wie Uckermark oder Mark Brandenburg nach.

3 Haushofer (1927, S. 136) fasst diese Entwicklung, die er allerdings unter den Vorzeichen von Oswald Spenglers Untergang des Abendlandes als Rückbildungsprozess begreift, noch differenzierter als eine „vom vagen Grenzraum zum Grenzsaum, von ihm zum Grenzstreifen, zum Grenzstrich, zur Grenzlinie“. 
grafen, Festungsbaumeister, Diplomaten und Juristen. Sie „entwickelten eine Vorstellung von Grenzen, für die einerseits die ,Erdung' der Grenze, ihre Zurückführung auf natürliche und naturräumliche Gegebenheiten im Vordergrund stand. Andererseits wurde hier die politische Herstellung, ja „,Machbarkeit‘ des Raums betont“ (Medick 1995, S. 215).

\subsection{Die Grenze der Neuzeit}

Merio Scattola (1997) hat dargestellt, dass ab der Mitte des 16. Jahrhunderts die Grenze zu einem staatsrechtlichen Thema wird. Es entstanden erste Theorien der öffentlichen Grenze, die zunächst noch unter vornaturrechtlichen Vorzeichen als partikulare oder privatrechtskonforme Grenzen innerhalb eines breiteren oder übergeordneten öffentlichen Bereiches aufgefasst, dann aber immer deutlicher von den privaten Grenzen abgehoben und auf der Basis der neu entstehenden Disziplin des Natur- und Völkerrechts begründet werden. Seit der zweiten Hälfte des 17. Jahrhunderts unterscheiden Dissertationen „mit großer Genauigkeit die private von der öffentlichen Grenze, wobei die ältere Vielzahl der öffentlichen Grenzen preisgegeben und eindeutig bestimmt wird, dass es nur eine einzige Staatsgrenze geben kann, weil sie auf der Einheit des Territoriums beruht“ (Scattola 1997, S. 64). Wie Scattola festhält, ist damit das Ende einer langen Entwicklung erreicht, mit dem die römische Auffassung „völlig auf den Kopf gestellt wird“ - während nämlich im Corpus iuris civilis die Grenze „nur eine privatrechtliche Geltung genießt, wird gerade diese privatrechtliche Bedeutung jetzt ausgeschlossen, und nur die öffentliche wird als ursprüngliche und eigentliche Grenze verstanden“ (ebd., S. 64f.).

Unter den Wörterbüchern ist es Johann Heinrich Zedlers Großes vollständiges Universal-Lexicon (1731-1754), das diesen kategorialen Paradigmenwechsel festhält und sprachlich konsequent umsetzt. Unter dem Lemma „Grentzen“ heißt es:

„Heutiges Tages wird dieser Unterschied in Acht genommen, daß man die Grentzen allein denen Land-Marcken, die man mit einem besonderen Namen Frontiere nennet, welche die Herrschafft und Gebiete von ein ander unterscheiden, und die Marck-Steine gemeiniglich nur denen privat-Gütern zugeeignet“ (Zedler 1731-1754, S. 831).

Verbunden mit dem Bedeutungszuwachs der Grenzterminologie setzt im 16. Jahrhundert auch in breiterem Umfang eine „ungemein fruchtbare Substantivcomposition“ ein (Hübner 1919/1935, Sp. 147), die vor allem auf die mit den (staats-)theoretischen Grenzreflexionen verbundenen Materialisierungen von Grenzen und die daran geknüpften Grenzpraktiken verweist:

„Am frühesten erscheinen naheliegende Bildungen wie Grenzbaum, -brunnen, -flusz, -kreuz, -mark (= Grenzzeichen), -rain, -stein, -wasser u.ä.; auch Grenzhaus, -schlosz, -stadt gehören schon dem 16. Jahrhundert an, ebenso Grenzbrief, -vertrag, selbst künstlichere Bildungen wie Grenzgedächtnis, -oberster. Auch persönliche Verbalsubstantiva setzen früh ein, vgl. grenzbereiter, -beschreiber, -bewahrer, -scheider, -verwahrer“ (ebd., Sp. 148).

Diese Grenzkomposita differenzieren dann im weiteren historischen Verlauf immer mehr aus, wobei laut Deutschem Wörterbuch für das 18. und 19. Jahrhundert die im 16. und 17. Jahrhundert noch eher seltenen Verbalsubstantiva auf -ung (wie etwa Grenzackerung, -bestätigung, -irrung, -scheidung, -setzung, -verschreibung, -verwahrung) besonders charakteristisch sind. 


\subsection{Nationalstaatliche Grenzen}

Die rasante Ausdifferenzierung des territorial-politischen Grenzvokabulars im 18. und 19. Jahrhundert steht vor allem im Zusammenhang mit der Herausbildung der modernen Nationalstaaten. Ein Vergleich der Einträge in Zedlers Großem vollständigem Universal-Lexicon (1731-1754) mit der von Johann Samuel Ersch und Johann Gottfried Gruber herausgegebenen Allgemeinen Encyclopädie der Wissenschaften und Künste (1818-1889) verdeutlicht die massive Verstaatlichung und Bürokratisierung der Grenze, die sich in einem umfangreichen Apparat von Registrierungsverfahren, Kontrollinstanzen sowie Grenzmarkierungs- und Grenzsicherungspraktiken niederschlägt, für die ein neues Vokabular geschaffen werden musste (vgl. Seweloh 1808) - die Allgemeine Enzyclopädie spricht im Artikel zum Lemma „Grenze“ u.a. von Grenzkommissionen, -polizei, -räthen, -kommissarien, -ämtern, -festungen, -städten, -dörfern, -protokollen, -rezeß, -vergleich, -register, -begehungen, -zügen, -beziehungen, -umgängen (vgl. Ersch/Gruber 1818-1889).

Mit der Bildung der Nationalstaaten verschärfen sich auch die Grenzstreitigkeiten und die im Medium symbolischer Identitätskonstruktionen vollzogenen Abgrenzungspolitiken, was sich auch in divergierenden Grenzvorstellungen niederschlägt. Markant ist die Differenz im Verständnis sogenannter natürlicher Grenzen zwischen Frankreich und Deutschland. Seit der zweiten Hälfte des 18. Jahrhunderts entwickelte sich hier vor allem bei eher ,staatsfernen Schriftstellern und Philosophen eine Vorstellung von den Grenzen der deutschen Nation als primär sprachlich-kulturell bestimmten Grenzen, die eng mit einem nationalen Kultur- und Identitätsverständnis verknüpft war. Als wesentliche (natürliche) Grenzen galten für diese Auffassung, wie dies in Frankreich der Fall war, nicht herrschaftlich-territoriale, politische Grenzen oder gar natürliche (physische) Grenzen, sondern vielmehr die sprachlich-kulturell und ethnisch bestimmten Grenzen (vgl. Medick 1995, S. 219). Prägnant kommt dieses Ineinandergreifen von nationaler Abgrenzung und ,innerer', sprachlich-kulturell und ethnisch bestimmter Grenzbildung in Johann Gottlieb Fichtes Reden an die deutsche Nation von 1808 zum Ausdruck: „Zuvörderst und vor allen Dingen: - die ersten, ursprünglichen und wahrhaft natürlichen Grenzen der Staaten sind ohne Zweifel ihre inneren Grenzen. [...] Aus dieser inneren, durch die geistige Natur des Menschen selbst gezogenen Grenze ergiebt sich erst die äußere Begrenzung der Wohnsitze, als die Folge von jener“ (Fichte 1808/1846, S. 460). Neben der Herstellung einer inneren Einheit bestand ein unmittelbares Ziel der deutschen Patrioten darin, die 1801 völkerrechtlich festgelegte Rheingrenze wieder nach Westen zu verschieben. Ein prominenter öffentlicher Stimmführer war Ernst Moritz Arndt, der in seiner 1813 erschienenen Schrift Der Rhein, Teutschlands Strom, aber nicht Teutschlands Gränze gegen die französischen Grenzvorstellungen (speziell die „angebliche Naturgränze“ des Rheins) polemisierte und das Konzept der natürlichen Grenze statt auf territoriale Aspekte auf die Sprache bezog: „Ich sage: die einzige gültigste Naturgränze macht die Sprache“ (Arndt 1813/1845, S. 6; Herv. i. O.). Und kurz darauf heißt es: „Aber Ströme sind nie Naturgränzen gewesen und können es auch nie werden“ (ebd., S. 9). 


\subsection{Grenzen in der politischen Geografie}

Die Vorstellung von den ethnisch bzw. sprachlich bestimmten natürlichen Staatsgrenzen bleibt in Deutschland auch über die Zeit der Reichsgründung 1871 hinaus präsent. Eine wirkmächtige Neukonfiguration erfährt sie im Rahmen der Politischen Geographie, die nach der Reichsgründung als neue universitäre Disziplin etabliert und gefördert wurde. Ihr wichtigster Vertreter ist Friedrich Ratzel, der als Begründer der Anthropogeografie gilt. Ratzel war von Haus aus Biologe und übertrug Charles Darwins Evolutionstheorie auf die gesellschaftlichen Umweltbeziehungen. Staaten begriff er als Organismen, die in einem beständigen Kampf ums Dasein, oder, wie Ratzel Darwins Formel spezifiziert, in einem beständigen Kampf um Raum stehen. In diesem Rahmen entwickelt er auch seinen Begriff der Grenze, die er „als peripherisches Organ des Staates“ versteht, das „sowohl der Träger seines Wachstums wie auch seiner Befestigung“ ist und „alle Wandlungen des Organismus des Staates mit[macht]“ (Ratzel 1896/1977, S. 42). Die politische Grenze wird damit zu einem „besonderen Fall“ des allgemeinen Begriffs der geografischen Grenze; ihre Behandlung wird deshalb „naturgemäß bei den Grenzen organischer Ausbreitungen zunächst Anschluss zu suchen haben“ (Ratzel 1892, S. 53). Ratzel dynamisiert damit den Begriff der Grenze, der bei ihm in enger Nachbarschaft mit imperialen Kampfbegriffen (wie Expansion, Invasion, Kolonisation) erscheint. Treibende Kräfte für die beständigen Grenzveränderungen sieht er im Auseinanderfallen von Lebensraum und Nahrungsraum bzw. von Staatsraum und Volkswachstum: je größer die Distanz zwischen Staatsgrenze und Lebensraum, umso verwundbarer ein Staat. Und umgekehrt gelte, dass ein Staat umso sicherer gegründet ist und seine Ausdehnung umso leichter gelinge, je näher der Lebensraum an den Grenzgebieten liege und je tiefer er in diesen verankert sei. Ältere Auffassungen von den natürlichen Grenzen aufnehmend, unterscheidet Ratzel zwischen abstrakten, politisch ausgehandelten linearen Grenzen und wirklichen, d.h. den dynamischen Volksentwicklungen Rechnung tragenden Grenzen. Die Völkergrenze ist für ihn ein Grenzsaum bzw. -raum. Damit wird der Begriff der Grenze zugleich verräumlicht, was sich auch im Verständnis der Grenze als „Kampfplatz“ ausdrückt (Ratzel 1901, S. 63). Die Ausdehnung des Staates unterliegt für Ratzel dem (Natur-)Gesetz der Zunahme der politischen Räume, dem sich der Staat nur bei Strafe seines Unterganges entziehen könne.

Ratzels Theorie ist im Zusammenhang des erstarkten nationalen Selbstbewusstseins und der Bemühungen im Deutschen Kaiserreich, in den Kreis der großen Kolonialmächte aufzurücken, zu sehen. Er selbst engagierte sich politisch im Vorstand der Leipziger Sektion der Deutschen Kolonialpolitik, im Alldeutschen Verband und im Deutschen Flottenverein. Mit seinem sozialdarwinistischen Raum- und Grenzdenken wurde er zur Leitfigur seiner Disziplin und zur Inspiration von Arbeiten zu verschiedenen Aspekten der Grenze (vgl. Schöne 1911; Penck 1916; 1917; Sieger 1917/18; 1921; 1926; Supan 1918; Maull 1919; 1925; Dix 1922a; 1922b; Sölch 1924a; 1924b; Vogel 1926). Ratzel und seine Schüler lieferten der späteren Geopolitik als spezifischer Ausgestaltung der Politischen Geographie ein theoretisches Fundament und wichtige Stichworte (wie z.B. den Begriff des Lebensraums), die für die nationalsozialistische Expansionspolitik in Dienst genommen werden konnten.

Die wichtigste Mittlerfigur war Karl Haushofer, der sich selbst als Schüler Ratzels bezeichnet. Obwohl durch Ratzel der Begriff der Grenze auch international zu einem zentralen Thema der Politischen Geographie aufgerückt und eine Vielzahl von Studien dazu erschienen war (vgl. Holdich 1916; La Pradelle 1928; Minghi 1977), reklamierte Haushofer in 
seiner 1927 erschienenen Schrift Grenzen in ibrer geographischen und politischen Bedeutung das Desiderat einer umfassenden Analyse der verschiedenen Dimensionen des Grenzbegriffs als Erster eingelöst zu haben. Er versteht seine Arbeit als Beitrag zur „geopolitischen [...] Grenzerziehung“ (Haushofer 1927, S. 108) des deutschen Volkes mit dem praktischen Ziel einer Revision der als „vergewaltigend“ angesehenen Grenzziehungen des Versailler Vertrages. Haushofer übernimmt Ratzels Verständnis der Grenze als „peripherisches Organ“ sowie tragende sozialdarwinistische Prämissen, dehnt dann aber das Grenzdenken auf nahezu sämtliche Lebensbereiche aus und versucht, eine Vielzahl verschiedener Grenzformen (z.B. Klimagrenze, Pflanzen- und Tiergrenzen), Sperrzonen (Kordon, Quarantäne, Korridor), Grenzfunktionen (z.B. Wehr-, Verkehrs-, Siedlungsgrenze) und Grenztypen zu erfassen. In einem Ordnungsvorschlag unterscheidet Haushofer fünf verschiedene Typen (vgl. ebd., S. 151-155; Herv. i. O.): erstens die „deutlich vorschreitenden, zum Greiforgan gewordenen, wehrgeographischen Angriffsgrenzen“, zweitens die „hoch organisierte, verkehrsdurchdrungene, jederzeit zum Vorschreiten entwickelte [...] Lauergrenze“, drittens „die Gleichgewichtsgrenze, die beiderseits gleich instinktsichere oder gleich bewusste Lebensformen zugleich scheidet und verbindet“, viertens „die Schutzgrenze im Abwehrstande“, fünftens „die entwehrte, der Durchdringung, der Unterwanderung preisgegebene“ Zersetzungsgrenze, „in die fremde Wachstumsspitzen und Verkehrskampfe eindringen“. Als Unterform der Gleichgewichtsgrenze sieht er die apathische, erstarrte, blutarme „Trägheitsgrenze oder auch Trägestaunngsgrenze“, der er wiederum die „übermäßig durchblutet[e], leicht erregt[e] und erregbar[e]“ tonische Reizgrenze gegenüberstellt.

Haushofers Arbeit bietet eine exorbitante Fülle neuer Grenzkomposita. Zum Vergleich: Während sich im 1935 erschienenen Deutschen Wörterbuch insgesamt 398 separate Einträge zu diversen Grenzbegriffen finden, umfasst Haushofers „Grenznomenklatur“ 275 Ableitungsformen (also annähernd 70 \% der Gesamtzahl des Deutschen Wörterbuchs), von denen im Deutschen Wörterbuch lediglich 73 verzeichnet sind - Haushofer kreiert also im Vergleich mit dem Deutschen Wörterbuch 202 neue Grenzkomposita, was einen Zuwachs von etwas über $50 \%$ bedeutet. Viele der Neologismen Haushofers setzen die in Deutschland verstärkt seit der Reichsgründung zu beobachtende Verbürokratisierung des Grenzvokabulars voraus und treiben sie weiter, und zwar nicht selten zu bizarren Blüten, die von den ideologischen Zügen dieses „staats-“ bzw. „grenzbiologischen“ (Haushofer 1927, S. 132, 29) Ansatzes zeugen Beispiele hierfür sind etwa die Ausdrücke Grenzdruckermittlung, -ferngefahr, -festigungserfahrung, -überschreitungskraft, -unterminierungsarbeit, -verantwortlichkeits-Bewusstsein, -verbitterung, -verschiebungsreiz, -volkstätigkeit.

\subsection{Expansionsgrenze: frontier}

Obwohl Haushofer sich wie auch schon Ratzel militant rassistischen Auffassungen verweigerte, ebnete er, wie ein Großteil der deutschen Vertreter der (Bio-)Geopolitik, mit seinem biologistischen und völkischen Grenzdenken der nationalsozialistischen Politik den Weg. Nach der Niederschlagung des Nationalsozialismus war dieser Strang des geopolitischen Denkens in Deutschland deshalb zu Recht kompromittiert. Die geopolitischen Grenzforscher in der Ratzel-Linie haben jedoch auch eine Reihe wichtiger Problemfelder markiert, die von den ausländischen Vertretern der Politischen Geographie aufgenommen und oft in kritischer Abset- 
zung von den deutschen Ansätzen weiterentwickelt wurden (vgl. Matznetter 1977). Im Blick auf Ratzel und die deutsche Geopolitik ist auch festzuhalten, dass das expansive Grenzdenken an der Wende zum 20. Jahrhundert kein Spezifikum der deutschen Theorieentwicklung war. Der österreichische Ökonom Joseph Schumpeter definierte im Jahre 1918 den Imperialismus als „die objektlose Disposition eines Staates zu gewaltsamer Expansion ohne angebbare Grenze“ (Schumpeter 1918, S. 3). Im amerikanischen Englisch steht vor allem der Ausdruck frontier für eine Expansionsgrenze par excellence (vgl. Böckler 2007). Er wird seit dem 17. Jahrhundert für die Bezeichnung der Siedlergrenze verwendet. Später wurde er dann zunächst auf die besondere Mentalität der amerikanischen Pioniere und dann schließlich auf die gesamte amerikanische Gesellschaft übertragen. Eine Leitfigur war Frederick Jackson Turner, der den Begriff der frontier in den 1890er-Jahren theoretisch entwickelt und damit zugleich den Grundstein für eine mythisierende Überhöhung der Siedlererfahrung als Kern der amerikanischen Identitätsbildung geliefert hat (vgl. Turner 1893/1920). 1914 übertrug Turner dann den Ausdruck auf „new frontiers of unwon fields of science, fruitful for the needs of the race; there are frontiers of better social domains yet unexplored“ (Turner 1914, S. 300) - eine Wendung zu „neuen Herausforderungen“ aller Art, die dann an wichtigen Wendepunkten der amerikanischen Geschichte von höchsten Repräsentanten des Staates aufgegriffen wurde: von Franklin D. Roosevelt 1932 im Zusammenhang mit der Weltwirtschaftskrise und des New Deal, von Vannevar Bush in seinem Bericht Science. The endless frontier (1945) an den Präsidenten der USA sowie von John F. Kennedy in einer Rede vom 15. Juli 1960, mit der er seine Nominierung zum Präsidentschaftskandidaten der Demokratischen Partei annahm. Die anhaltende Relevanz und internationale Ausstrahlung dieser „Mobilisierungsmetapher“ (Bredow 2014, S. 92) zeigt sich daran, dass die Europäische Kommission im Jahre 2005 den programmatischen Leitbegriff der Grundlagenforschung basic research durch den Begriff frontier research ersetzt hat (vgl. Flink/Kaldewey 2018). Auch das 1902 geprägte Schlagwort von Amerika als ,Land der unbegrenzten Möglichkeiten“ reflektiert die im frontier-Begriff festgehaltene Expansionskultur (vgl. Grossmann 1994; Petersen 1996).

\section{3 Übertragungen des Grenzbegriffs}

Die Ausbreitung der Grenzterminologie seit dem 16. Jahrhundert stand in einem engen Zusammenhang mit der Etablierung des modernen Territorialstaates und war semantisch entsprechend auf politisch-geografische Aspekte bezogen. Parallel dazu lassen sich vor allem bei Reformatoren und Humanisten übertragene Gebrauchsweisen erkennen, die sich von den räumlichen Bezügen lösten. Ein wichtiges Feld bilden speziell seit dem 16. Jahrhundert die Diskussionen um die Ausdehnung und Grenzen des Universums im Schnittfeld von Theologie, Philosophie, Mathematik und Astronomie. In diesen Debatten formte sich eine gänzlich neue Bedeutungsdimension von Grenze heraus, die dann vor allem seit dem 18. Jahrhundert zunehmende Verbreitung findet: „Während der Begriff der Grenze im ursprünglichen Sinne auf der Vorstellung eines Raumes diesseits oder jenseits der Scheidelinie fußt“", entwickelt sich nun „ein Gebrauch, der von dem Raum jenseits der Grenze mehr oder weniger absieht und das Wort [Grenze] so den Bedeutungen ,Schranke, Abschluss, Ziel, Ende“ nähert“ (Hübner 1919/1935, Sp. 134).

Indem die andere Seite der Beziehung nicht mehr ausdrücklich benannt wird, ist sie durch den Ausdruck nun selbst nicht mehr positiv, sondern nur noch durch Ausschluss definiert, womit offensichtlich auch die Symmetrie der Beziehung verloren geht (vgl. Böckler 2003, S. 211). Es 
ändert sich durch diese Übertragung die logische Binnenstruktur des Begriffs - aus der Grenze zwischen wird die Grenze von.

Eine Konsequenz dieser neuen Bedeutungsdimension ist der prominent bei Gottfried Wilhelm Leibniz zu beobachtende Versuch, zwischen Grenze und Schranke zu differenzieren (vgl. Fulda 1974, S. 875). Ein spezieller Beitrag von Leibniz zur Diskussion um den mathematischen Grenzbegriff ist seine Infinitesimalrechnung, mit der unendlich kleine (infinitesimale) Intervalle einer Funktion widerspruchsfrei beschrieben werden können. Die Bedeutung dieser neuen Dimension von Grenze zeigt sich in den Wörterbüchern und Enzyklopädien des 18. und 19. Jahrhunderts, die oft erstaunlich umfangreiche Beiträge zu diesem mathematischen Bedeutungsspektrum von „Grenze“ enthalten, das vom politisch-geografischen Bedeutungsstrang deutlich abgehoben wird. Im Verlauf der Entwicklung der Mathematik bildet sich eine ausdifferenzierte fachspezifische Terminologie heraus, die dann ihrerseits zum Reservoir weiterer Metaphorisierungen wird: zu den neuen Begriffen gehören u.a. Grenzwert, -beziehung, -form, -lage, -winkel, -zahl, -ziffer, -begriff, -bestimmung, -punkt oder das Begriffspaar Supremum und Infimum zur Bezeichnung der kleinsten oberen bzw. der größten unteren Schranke bei der Untersuchung halbgeordneter Mengen. Als übergreifende Klammer der beiden Entwicklungen des Grenzbegriffs seit dem 16. Jahrhundert ließe sich der speziell auch am Wandel der kartografischen Darstellungen verfolgbare Prozess neuzeitlicher Rationalisierung des Lebens verstehen, den auf dem Feld der Politik am reinsten die Grenze in Form einer geometrischen Linie verkörpert (vgl. Schneider 2006; Barth 2018).

\subsection{Grenze als erkenntniskritische Kategorie}

Die Mathematik ist aber nur ein, wenngleich wichtiges Feld, auf das der Grenzbegriff im Zuge der Ausbreitung der bürgerlichen Gesellschaft übertragen wird. Speziell seit dem 18. Jahrhundert dehnt sich der Gebrauch des Grenzbegriffs auf nahezu jedes Feld aus. Die Übertragungsdynamik erfolgt dabei keineswegs beliebig, sondern lässt verschiedene feldspezifische Muster, Dynamiken und Übertragungslogiken erkennen, die sich bestimmten sozial- und kulturhistorischen Entwicklungen zuordnen lassen. Verallgemeinert gesprochen, wird spätestens mit der Aufklärung Grenzbewusstsein als Verfahren der Selbstreflektion und Erkenntniskritik methodisch relevant. In besonderer Weise gilt das für die Philosophie. Bereits René Descartes (1626-28/1972, S. 28) erörtert am Beginn der neuzeitlichen Philosophie die „Grenzen der Erkenntniskraft“ (ingenii limites), und speziell mit Immanuel Kant wird der Rückgriff auf den Grenzbegriff als erkenntniskritische Kategorie verbindlich. Philosophie, heißt es in der Kritik der reinen Vernunft, bestehe darin, „seine Grenzen zu kennen“. In den Prolegomena zu einer jeden künftigen Metaphysik, die als Wissenschaft wird auftreten können (1783) handeln die Paragrafen 57 bis 60 „Von der Grenzbestimmung der reinen Vernunft“. Verstärkt seit dem 19. Jahrhundert forderten die Entwicklungen der Naturwissenschaften Revisionen epistemologischer Grenzbegriffe heraus (vgl. Du Bois-Reymond 1873; Rickert 1896/1902). An den metaphorischen Dimensionen der Grenzbegriffe lassen sich zugleich die kulturellen und sozialen Einbettungen und Voraussetzungen der erkenntniskritischen Reflexionen ablesen, so etwa schon am vorkritischen Werk Kants, wenn es heißt, die Metaphysik sei „eine Wissenschaft von den Grenzen der menschlichen Vernunft, da ein kleines Land jederzeit viel Grenze hat, 
überhaupt auch mehr daran liegt, seine Besitzungen wohl zu kennen und zu behaupten, als blindlings auf Eroberungen auszugehen“ (Kant 1766/1912, S. 368).

Bei Georg Wilhelm Friedrich Hegel bekommt der Grenzbegriff dann bereits eine weitaus existenziellere Bedeutung, wenn er, in Absetzung sowohl von einer bloß erkenntniskritischen wie auch von mathematischen Bedeutungen, als eine wesentliche Dimension von Sein dynamischqualitativ bestimmt wird:

„Die Negation ist im Dasein mit dem Sein noch unmittelbar identisch, und diese Negation ist das, was wir Grenze heißen. Etwas ist nur in seiner Grenze und durch seine Grenzen das, was es ist. Man darf somit die Grenze nicht als dem Dasein bloß äußerlich betrachten, sondern dieselbe geht vielmehr durch das ganze Dasein hindurch. Die Auffassung der Grenze als einer bloß äußerlichen Bestimmung des Daseins hat ihren Grund in der Verwechslung der quantitativen mit der qualitativen Grenze. Hier ist zunächst von der qualitativen Grenze die Rede“ (Hegel 1830/1970, S. 197, Herv. i. O.).

In seiner Wissenschaft der Logik (1812/16) hatte Hegel eine spezifische Form des Hindurchgehens der Grenze durch das ganze Dasein in der „schlechten Unendlichkeit [...] des Progresses des Quantitativen ins Unendliche“ gesehen. Gemeint ist damit das „fortgehende Überfliegen der Grenze“, das in der bürgerlichen Gesellschaft „für etwas Erhabenes und für eine Art von Gottesdienst gehalten“ werde (Hegel 1812-16/1969, S. 264, Herv. i. O.).

Karl Marx verknüpft dann im Rahmen seiner Kritik der politischen Ökonomie (185758/1974) die erkenntnis- wie auch die seinskritischen Dimensionen des Grenzbegriffs, wenn er sich im Zusammenhang von Darstellungsfragen der Grenzen der Dialektik (vgl. Haug 2001) versichert und zugleich die von Hegel beschriebene Expansionslogik des fortgehenden Grenzüberfliegens als Bewegungsprinzip der vom Kapitalverhältnis dominierten modernen Gesellschaft dechiffriert. „Die Tendenz[,] den Weltmarkt zu schaffen[,] ist unmittelbar im Begriff des Kapitals selbst gegeben. Jede Grenze erscheint als zu überwindende Schranke“ (Marx 1857-58/1974, S. 311, Herv. i. O.) Ganz ähnlich und stark an Hegels Formulierung erinnernd, sagt Carl v. Rodbertus-Jagetzow von den „Capitalien“, dass sie geradezu „die Beweglichkeit selbst“ sind und es vermögen, „sich in alle Formen umzusetzen, alle nationalen Grenzen zu überfliegen, sich zu ungeheuren Summen zusammenzuschließen “ (Rodbertus-Jagetzow 1869, S. V). Marx’ Kritik der politischen Ökonomie gibt als kritische Theorie der modernen Gesellschaft zugleich eine Erklärung für die seit der Neuzeit zu verzeichnende Expansion des Grenzbegriffs an die Hand - der Begriff drängt sich auf, weil die an der Schwelle zur Neuzeit sich herausbildende Gesellschaft als Ganze von einer Dynamik beherrscht ist, die bestehende Grenzen permanent in Frage stellt, unterläuft, überschreitet und verschiebt. Marx’ kritische Analyse dringt bis zu dem Punkt vor, wo die historischen Grenzen dieser Gesellschaftsform selbst aufscheinen. Die Marx'sche Theorie der modernen Gesellschaft ist damit ein Paradefall einer weiteren wesentlichen Entwicklung des Grenzbegriffs, die sich seit dem letzten Drittel des 18. Jahrhunderts anbahnt und im 19. Jahrhundert rasch an Bedeutung gewinnt: die Ausweitung auf die zeitliche Dimension und damit seine Temporalisierung. Die Grenze wird so von einer Linie oder Zone der Scheidung im Raum zu einer Grenze der Zeit, die verschiedene Epochen, Perioden, Weltalter etc. voneinander trennt. 


\section{2 Übertragungen der Aufklärung}

Neben und im engen Kontakt mit der Entwicklung der erkenntniskritischen Dimensionen des Grenzbegriffs auf dem Gebiet der Philosophie entwickelt sich speziell seit der Aufklärung ein Grenzbegriff zur Reflexion der Zuständigkeiten und Formen bestimmter Wissensbereiche. Entdeckt die Aufklärung mit der Historizität der Begriffe zugleich das Erfordernis einer Reflexion ihrer Reichweite und ihres Umfangs, so wiederholt sich das in Bezug auf die disziplinären Organisationsformen des Wissens, die seit dem letzten Drittel des 18. Jahrhunderts in Bewegung geraten und neu begründet werden müssen. Ein seit dieser Zeit notorisches Feld ist die Kunsttheorie, für die u.a. Gotthold Ephraim Lessing mit seiner Schrift Laokoon oder über die Grenzen der Mablerey und Poesie (1766) Maßstäbe gesetzt hat. Georg Gottfried Gervinus reflektiert dann schon verallgemeinernd „jenes von Lessing begriffene Bestreben der Zeit, in alle geistige Thätigkeiten, in die Gattungen der Dichtung, in die Grenzorte der Kunst und Wissenschaft [...] eine reine Scheidung zu bringen“ (Gervinus 1842, S. 406). Lessings Ansatz inspirierte eine Vielzahl anderer Versuche, die Grenzen verschiedener Wissenschaften, Gattungen, Künste oder zwischen Wissenschaft und Kunst auszuloten und die nun öfter beklagten „Grenz(en)verwirrungen“ aufzuklären - bis zum Beginn des 19. Jahrhunderts entsteht so eine Fülle von Schriften zu den Grenzen der Musik und Poesie, der Malerei, der Kunst, der Mythologie, der Dichtung u.a.m. Eine von Lessing in seiner Hamburgischen Dramaturgie (1767/69) kommentierten Abhandlung von Richard Hurd mit dem Titel Über die verschiednen Gebiete des Drama verweist ähnlich wie Gervinus’ Begriff des Grenzorts oder der Grenzbegriff des frühen Kant auf den territorialen Hintergrund und Bildspender dieser Übertragungen.

Dass es bei diesen Gebietsabsteckungen immer auch um Machtverhältnisse und Disziplinierungen geht, zeigt sich besonders deutlich an einem weiteren wichtigen Feld, auf dem der Grenzbegriff im übertragenen Sinne speziell seit der Aufklärung an Bedeutung gewinnt: dem der politischen und juristischen Theorie des Staates. Ein Standardthema ist die Bestimmung der Grenzen seiner Gewalt und Befugnisse. Ein Beispiel hierfür ist Wilhelm von Humboldts Arbeit Ideen zu einem Versuch, die Gränzen der Wirksamkeit des Staats zu bestimmen, die im Jahre 1792 entstanden ist, aber zunächst verboten und erst im Jahre 1851 vollständig veröffentlicht wurde. Anton Kurz, Regierungsrat und Abgeordneter zur zweiten Kammer der Stände des Königreichs Bayern meinte 1821 im Hinblick auf die Literatur der Zeit feststellen zu können, dass „der Grundsatz“ von „allen Staatsrechtlehrern und Staats-Männern als richtig anerkannt“ sei, „ohne Trennung der Gewalten, ohne genaue Bezeichnung der Grenze ihres Wirkungskreises“ könne „keine vollständige Garantie für die Rechte der Staatsglieder“ bestehen (Kurz 1821, S. 22). Ein damit verbundenes Thema bilden die Diskussionen um die Grenzen der Pressefreiheit. Henrik Steffens zufolge erkenne jeder „gebildete Staat“ an, „daß die Grenze seiner Gewalt da sei, wo das Geistige angeht“ (Steffens 1808-09/1956, S. 324). Vorher hatte schon Martin Wieland die Auffassung vertreten, es stände der Gesellschaft übel an, „der Aufklärung [...] unnatürliche Grenzen setzen zu wollen, da sie doch, vermöge der Natur des menschlichen Geistes ebenso grenzenlos ist als die Vollkommenheit, wozu die Menschheit mit ihrer Hülfe gelangen kann und soll!“ (Wieland 1788/1930, S. 229). Eine weitere „ebenso natürliche Folge der Aufklärung“ erblickt er in dem Umstand, dass, „,je weiter die Grenzen unserer Kenntnisse hinaus gerückt werden“, desto weiter dehne sich „auch der Kreis des Möglichen“ aus (Wieland 1781/1928, S. 326). 


\subsection{Ausdifferenzierung fachspezifischer Grenzvokabulare}

Neben solchen Zentralthemen finden sich Übertragungen des Grenzbegriffs auf alle erdenklichen anderen Bereiche - so ist von den Grenzen des Anstands, des guten Geschmacks, der Sittlichkeit usw. die Rede. Wie oben schon anhand der Mathematik gezeigt wurde, bilden sich mit der gesellschaftlichen Ausdifferenzierung und der Entstehung neuer Disziplinen und Forschungsfelder verschiedene fachspezifische Grenzvokabulare aus. Ein Beispiel hierfür ist die Politische Ökonomie bzw. die Nationalökonomie. In der zweiten Hälfte des 19. Jahrhunderts bildete sich gegen die von der klassischen Politischen Ökonomie von Adam Smith über David Ricardo bis hin zu John Stuart Mill sowie von Karl Marx vertretene objektive (Arbeits-)Wertlehre die subjektive Wertlehre heraus. In ihrem Rahmen entstand die sogenannte Grenznutzenschule. Ihren Namen erhielt sie nach dem von Friedrich von Wieser (1884, S. 128) als Äquivalent zu Hermann Heinrich Gossens „Wert des letzten Atoms“ und als deutsches Komplement zu angelsächsischen oder französischen Begriffen bzw. Theoremen wie final degree of utility, terminal utility oder intensité du dernier besoin satisfait eingeführten Begriff des Grenznutzens. Dieser Begriff bezeichnet „den Nutzen, den der letzte zur Verfügung stehende Teil des Vorrates an einem Gute für ein bestimmtes wirtschaftendes Subjekt hat; nach ihm bemiszt sich der Wert einer Mengeneinheit des Gutes für das betreffende Subjekt“ (Hübner 1919/1935, Sp. 172). Die Vertreter dieser Lehre werden Grenzwerttheoretiker genannt. Auf der Grundlage des Theorems vom Grenznutzen wurden innerhalb dieser Schule dann weitere ökonomische Grenzbegriffe entwickelt, wie etwa „Grenzarbeiter“, „Grenzunternehmung“ oder „Grenzboden“.

Die in der Aufklärung entwickelte Vorstellung von der „grenzenlosen“ bzw. „unbegrenzten “4 Vervollkommnungsfähigkeit des Menschengeschlechts ruht auf geschichtsphilosophischen Prämissen, die im letzten Drittel des 19. Jahrhunderts an Plausibilität zu verlieren begannen. Hatte Marx bereits die historischen Schranken der modernen Gesellschaft aufgewiesen, so sind es am Beginn des 20. Jahrhunderts vor allem marxistische Theoretiker, die das Ziel verfolgen, im Anschluss an Marx möglichst exakt die objektiven Grenzen des Kapitalismus, den Endpunkt von dessen Entwicklung zu bestimmen. Rosa Luxemburg (1913/1975) vertrat die These, dass mit der Durchkapitalisierung der nichtkapitalistischen Länder der Kapitalismus notwendig zusammenbrechen müsse, da er keine äußeren Absatzgebiete mehr vorfinde. Henryk Grossmann legte das von Marx postulierte Gesetz vom tendenziellen Fall der Profitrate zum ersten Mal systematisch als Theorie des unausweichlichen Zusammenbruchs des Kapitalismus aus. Die Leitfrage seines Buches,

„ob der vollentwickelte Kapitalismus als ausschließliches und allgemeines, nur auf sich selbst angewiesenes Wirtschaftssystem imstande ist, den Reproduktionsprozess auf einer fortschreitend sich erweiternden Basis schrankenlos zu entfalten, oder aber ob für diese Erweiterung nicht etwa irgendwelche unüberschreitbare Grenzen bestehen " Grossmann 1929, S. VIII)

beantwortet er nach Art eines mathematischen Beweises mit der These, dass für die Kapitalakkumulation „eine exakt bestimmbare Maximalgrenze gezogen“ sei, „weil die Höchstquote der erzielbaren Mehrwertmasse exakt gegeben ist“ (ebd., S. 523). Infolge mangelnder Kapitalverwertung tritt für ihn „notwendig das Ende der Kapitalakkumulation ein“ (ebd., S. 164).

4 Zur Auseinanderentwicklung dieser anfänglich synonymen Wörter vgl. das Lemma „grenzenlos“ im Deutschen Wörterbuch der Gebrüder Grimm. 
$\mathrm{Zu}$ den semantischen Variationen seiner Zusammenbruchstheorie gehören Begriffe wie „Entwicklungsgrenze“, „Akkumulationsgrenze“, „Zusammenbruchsgrenze“ oder „Verwertungsgrenze“, die ebenso wie seine Wendung vom „Entropiegesetz der Kapitalakkumulation“ (ebd., S. 190) auf den Einfluss naturwissenschaftlicher Methoden hindeuten. John Maynard Keynes spricht in ähnlichem Zusammenhang von der „Grenzleistungsfähigkeit des Kapitals“ (englisch marginal efficiency of capital; Keynes 1936/1983, S. 185).

Am Beginn des 20. Jahrhunderts spielt der Grenzbegriff auch in der Lebensphilosophie und der Soziologie eine Rolle, prominent etwa und in verschiedenen Zusammenhängen und Variationen bei Georg Simmel. In seinen Untersuchungen über die Formen der Vergesellschaftung konstatiert er, der Begriff der Grenze sei „in allen Verhältnissen von Menschen untereinander äußerst wichtig, wenngleich sein Sinn nicht immer ein soziologischer ist“ (Simmel 1908/1992, S. 696). Gegen naturalistische Grenzauffassungen betont er den Konstruktionscharakter, die gesellschaftliche Vermitteltheit von Grenzen: „Die Grenze ist nicht eine räumliche Tatsache mit soziologischen Wirkungen, sondern eine soziologische Tatsache, die sich räumlich formt “ (ebd., S. 697). Und an anderer Stelle heißt es, dass „,jede Grenze [...] ein seelisches, näher: ein soziologisches Geschehen" sei (ebd., S. 699). In der späten Arbeit Lebensanschauung nimmt Simmel die soziologische Bedeutung zurück und wertet sie als eine anthropologische, wenn er den Menschen als ein „Grenzwesen, das keine Grenze hat", bestimmt (Simmel 1918/1999, S. 218) und ganz allgemein das Leben „als Einheit von Grenzsetzung und Grenzüberschreitung“ betrachtet (ebd., S. 230). Parallele Begriffe wie „revolutionär“ oder „schöpferisch" zeigen aber an, dass dieser lebensphilosophische Ansatz Bestimmungen naturalisiert, die Marx schon als dezidiert gesellschaftliche ausgewiesen hatte. Ganz ähnlich verhält es sich mit dem von Karl Jaspers im Jahre 1919 wohl in Reaktion auf die Erfahrung des Ersten Weltkrieges geprägten und seitdem immer wieder aufgegriffenen Begriff der Grenzsituation. Grenzsituationen, auch Grundsituationen oder letzte Situationen genannt, sind unvermeidliche Widerfahrnisse (wie beispielsweise Tod, Zufall, Schuld und die Unzuverlässigkeit der Welt), bei denen der Mensch auf seine Grenzen stößt und sein Scheitern erfährt. Für Jaspers ist nun entscheidend, wie der Mensch sich in diesen Grenzsituationen verhält, ob er sie verdrängt und ihnen ausweicht oder ob er sie nutzt, um zu seiner Existenz durchzubrechen. „Grenze bezeichnet dann nicht mehr bloß die endgültige Beschränkung, sondern zugleich die Stelle, an der das Dasein auf Transzendenz hin durchsichtig wird und somit aus möglicher in wirkliche Existenz umschlägt“ (Saner 1974, S. 878). „Grenzsituationen erfahren und Existieren ist dasselbe" (Jaspers 1932, S. 204, Herv. i. O.). Hans Blumenberg sieht in der Figur des Scheiterns „eine Metapher der Grenzerfahrung, die erst mit der nachlebensphilosophischen Existenzphilosophie“ (Blumenberg 1987, S. 123) die historische Bühne betritt.

Mit dem Anwachsen sozialer Antagonismen wird auch der Grenzbegriff vermehrt zu einem Kampfkonzept. Ab Mitte der 1920er-Jahre entstehen zeitlich parallel und oft auch in Reaktion auf die marxistischen Zusammenbruchstheorien kulturkritische Ansätze, die zwar oft immer noch auf lebensphilosophischen, anthropologischen oder naturalistischen Prämissen beruhen, aber den Grenzbegriff wieder verstärkt mit sozialkritischen Gehalten verbinden. Oswald Spengler sieht im Anschluss an Friedrich Nietzsche in der Geschichte einen „Wille[n] zur Macht“ am Werk, „der aller Grenzen von Zeit und Raum spottet, der das Grenzenlose, das Unendliche zum eigentlichen Ziel hat“ und „sich ganze Erdteile [unterwirft]“ (Spengler 1931, S. 62). An diesem Willen werde die abendländische Zivilisation zerbrechen. Anklänge an das Zusammenbruchstheorem von Rosa Luxemburg finden sich in Jaspers' Die geistige Situation 
der Zeit (1931/1964). Darin wird konstatiert, dass der Planet „zum erstenmal [...] der eine umfassende Wohnplatz des Menschen“ geworden und damit „die äußere Eroberungsbewegung [...] an ihre Grenze gestoßen [ist]; die sich ausbreitende Bewegung trifft gleichsam im Rückstoß auf sich selbst“ und lasse so „die Grenze der Daseinsordnung“ (Jaspers 1931/1964, S. 17, 37) hervortreten. Der Begriff der Grenze nimmt in diesen Diskursen, ähnlich wie bei den marxistischen Vertretern, die Bedeutung des Endes des Kapitalismus an und dient zur Untersuchung der genaueren Formen und Gründe dieses Endes. Helmuth Plessner hat in seiner 1924 veröffentlichten Arbeit Grenzen der Gemeinschaft. Eine Kritik des sozialen Radikalismus (1924/2002) den Grenzbegriff in umgekehrter Richtung gegen die linke wie rechte Gesellschaftskritik gekehrt und damit einen soziologischen Grenzbegriff entwickelt, der bis heute wirkmächtig ist. Aber auch seine später entwickelte philosophische Anthropologie sieht die „prometheische Kultur“ der Moderne „unter dem Gesetz der Grenzenlosigkeit“ stehen, weshalb sich das Problem der Unmenschlichkeit „mit größerer Dringlichkeit als früher“ (Plessner 1967/2003, S. 334, 328) und in wachsender Schärfe stelle.

\subsection{Grenzen und Extreme}

Ein hervorstechender Zug des Grenzdenkens der 1930er-Jahre ist die Verknüpfung des Begriffs der Grenze mit anderen (oft temporalen) Grenzbegriffen wie denen des Rekords, des Tempos, der Front oder des Extrems, die ihrerseits den Begriff der Grenze historisch profilieren und feldspezifisch neu bestimmen. In diesem Sinne sieht es Ernst Jünger 1932 in seinem Essay Der Arbeiter. Herrschaft und Gestalt als Signatur seiner Zeit an, „fortwährend über die äußersten Grenzen der Leistungsfähigkeit unterrichtet zu sein“ (Jünger 1932/1982, S. 146). Im Deutschen Wörterbuch wird dieses Bedeutungssegment als ein spezifischer Wortgebrauch von Grenze beschrieben, „der die Annäherung eines Zustandes an sein Extrem bezeichnet“ und sich darin mit der Bedeutung von „alle Grenzen überschreitend, maßlos, rein quantitierend“ berührt (Hübner 1919/1935, Sp. 155). Dieser Gebrauch, der sich überhaupt erst in der zweiten Hälfte des 18. Jahrhunderts entwickelt, hat seitdem beständig an Bedeutung wie auch an existenzieller Wucht gewonnen.

Ein neuer Grenzbegriff, der die traumatischen Erfahrungen der Konzentrationslager reflektiert, ist der 1943 von Bruno Bettelheim eingeführte Begriff der Extremsituation, der in einem Aufsatz mit dem Titel Die äußerste Grenze entwickelt wird (vgl. Bettelheim 1943/1982).

„Wir befinden uns dann in einer Extremsituation, wenn wir in eine Lage hineinkatapultiert werden, in der unsere alten Anpassungsmechanismen und Wertvorstellungen nicht mehr helfen, ja wo sogar einige von ihnen unser Leben gefährden, anstatt es wie früher zu schützen. In dieser Situation werden wir sozusagen unseres ganzen Abwehrsystems beraubt, und wir werden so weit zurückgeworfen, daß wir - der Situation gemäß - neue Einstellungen, Lebensweisen und Wertvorstellungen entwickeln müssen“ (ebd., S. 20).

Seit den 1950er-Jahren lässt sich eine zunehmende Verbindung des Grenzbegriffs mit der Technikentwicklung beobachten. Ein wichtiges Feld ist das der Auseinandersetzungen mit der Atombombe und den neuen Massenvernichtungsmitteln. Ein einflussreicher philosophischer Interpret ist Günther Anders, der die conditio humana grundlegend verändert sieht und erkenntniskritische Konsequenzen daraus zieht. 
„Eine Kritik der Grenzen des Menschen, also nicht nur der seiner Vernunft, sondern der Grenzen aller seiner Vermögen (der seiner Phantasie, seines Fühlens, seines Verantworten[s] usf.) scheint mir heute, da sein Produzieren alle Grenzen gesprengt zu haben scheint, und da diese spezielle Grenzsprengung die noch immer bestehenden Grenzen der anderen Vermögen umso deutlicher sichtbar gemacht hat, geradezu das Desiderat der Philosophie geworden zu sein“ (Anders 1956/2002, S. 18, Herv. i. O.).

Im Zusammenhang mit den Atombombentests ergeben sich seit den 1950er-Jahren intensive Diskussionen um die zulässigen Obergrenzen radioaktiver Belastungen - ein Thema, das dann mit der Entstehung der Ökologiebewegung seit Ende der 1960er-Jahre eine allgemeinere Bedeutung gewinnt und sich auf eine Fülle sogenannter Schadstoffe bezieht. Debatten um Grenzwerte, Obergrenzen, Höchstbelastungsgrenzen, ihre Konstitution, Einhaltung, Kontrolle und Ahndung bilden seitdem ein durchgängiges Politikum, dessen Bedeutung beständig zu wachsen scheint, wie neuere Diskussionen um Schadstoffemissionen oder Treibhausgase und die darauf bezogenen Grenzbegriffe (Stoppfunktion, Leitplanke, Korridor, Tempolimit u.a.) zeigen.

\subsection{Grenzbegriffliches Neuland: die Politische Ökologie und die Grenzen im Globalisierungsdiskurs}

Die Entstehung der Ökologiebewegung markiert dann wohl auch den tiefsten Einschnitt in der jüngeren Diskursgeschichte zum Thema und Begriff der Grenze, den die klassische Pionierarbeit Die Grenzen des Wachstums (Meadows et al. 1973) signalgebend im Titel trägt. Das Buch wurde in kürzester Zeit zu einem Weltbestseller und richtete das ökologiepolitische Denken neu aus, wobei der Grenzbegriff eine Schlüsselrolle spielte (vgl. Mansholt 1974; Peccei/Siebker 1974; Illich 1975). Die Hauptthese ist, dass das moderne Wirtschaftssystem mit seinem Wachstumsimperativ zwangsläufig dazu tendiert, über gegebene Naturgrenzen hinauszuschießen, was die Gefahr eines „Zusammenbruchs“ impliziere. Damit kehrt die speziell vom marxistischen Denken bearbeitete Problematik der systemischen Grenzen des Kapitalismus zurück, die jetzt aber nicht mehr in immanenten Faktoren, sondern in den (externen) natürlichen Bedingungen gesehen werden. Für den Fall eines Anhaltens der Entwicklungstrends prognostizieren die Autoren das Erreichen der „absoluten Wachstumsgrenzen auf der Erde im Laufe der nächsten 100 Jahre“ (Meadows et al. 1973, S. 17). Sie sehen die Menschheit damit an einem „Wendepunkt der Geschichte“ (ebd., S. 174) stehen - eine Formel, die im Bunde mit der zäsuralen Wendung des „zum ersten Mal“ für die frühe Rezeption prägend wird (vgl. Mesarović/Pestel 1974). Herbert Gruhl (1975, S. 225) spricht von einer „kopernikanischen“ bzw. „planetarischen Wende“ (ebd., S. 226) und zieht als Lehre den Schluss, dass das neue Denken „von den Grenzen dieses Planeten ausgehen“ muss (ebd.). Mit der Wendebestimmung geht zugleich eine neue Betrachtung der Vergangenheit einher, als deren „Kulturidol“ Gruhl „das Prinzip des Kampfes gegen Grenzen“ sieht (ebd.). Ganz ähnlich war es für Erhard Eppler,

„das Pathos der europäischen Geschichte, zumindest seit Beginn der industriellen Revolution, wenn nicht schon seit der Renaissance, die Überwindbarkeit von Grenzen immer neu zu demonstrieren: Grenzen des Wissens und Erkennens, Grenzen der Leistung, Grenzen der Geschwindigkeit, Grenzen der Produktivität und der Produktion, Grenzen des Raumes, schließlich Grenzen des Erdballs selbst“ (Eppler 1975, S. 11). 
Entsprechend besteht für ihn der „historische Wendepunkt“ im (geforderten) Übergang „von einem Zeitalter der Grenzüberwindung zu einem Zeitalter der Grenzbestimmung “ (ebd., S. 22). War schon die Temporalisierung der Grenze, wie sie sich etwa in der um 1800 entstehenden Rede von der Grenze der Zeiten manifestiert, eine semantische Innovation, so gewinnt die Temporalisierung eine neue Qualität, wenn das jüngste Zeitalter selbst als eines der permanenten Grenzüberschreitung charakterisiert wird.

Als eine Konsequenz ihrer Befunde forderten die Autoren der Grenzen des Wachstums, „neue Denkgewohnheiten zu entwickeln, die zu einer grundsätzlichen Änderung menschlichen Verhaltens und damit auch der Gesamtstruktur der gegenwärtigen Gesellschaft führen “ (Meadows et al. 1973, S. 170). Ihre Anregung, „bessere Einsichten in die Ursachen [...] für die Grenzen des Wachstums zu gewinnen“ (ebd., S. 80), wurde in der Folgezeit aufgenommen und führte zur Reduktion des zunächst noch beträchtlichen Mangels „an grundlegenden Daten und selbst an einem elementaren Vokabular im Zusammenhang mit der [...] Überschreitung von Grenzen“ (Meadows et al. 2006, S. XVI). Die 1992 präsentierte Nachfolgestudie Die neuen Grenzen des Wachstums, die mit dem Kapitel „Grenzüberschreitung“ beginnt, präsentiert ein Glossar, in dem wichtige Grenzbegriffe der ökologischen Systemforschung definiert werden. Zum Stichwort „Grenzen“ heißt es: „Im Sinne dieses Buches sind Grenzen nicht als geographische Begrenzungen zu verstehen, sondern allgemein als Höchstbelastungen, die man der Umwelt aufbürden kann, ohne ihre lebenswichtigen Funktionen nachhaltig zu stören “ (Meadows et al. 1993, S. 299). „Grenzüberziehung“ wird als „zeitweiliger Zustand, bei dem für die Dauer nicht überschreitbare Grenzen überschritten sind“, definiert.

„Grenzüberziehung wird verursacht durch Verzögerungen oder fehlerhafte bzw. falsch verstandene Informationen über Systemgrenzen, die es unmöglich machen, das System in den auf Dauer erträglichen Grenzen zu halten. Grenzüberziehung kann auch als Ergebnis einer zu hohen Wachstumsrate auftreten, so dass das hohe Moment der Veränderung ein Hinausschießen über Grenzen unvermeidbar macht. Grenzüberziehung muss durch Systembeschränkung rückgängig gemacht werden, da sonst die Gefahr eines Zusammenbruchs droht" (ebd.).

Die Ausdifferenzierung des ökologischen Grenzvokabulars und die Entwicklung neuer quantitativer Maße für die Diskussion der Grenzüberschreitung erfolgt auf breiterer Basis ab Mitte der 1990er-Jahre. In ihrer neuen Arbeit Grenzen des Wachstums. Das 30-Jahre-Update heben die Autoren besonders das von Mathis Wackernagel und Kollegen entwickelte Konzept des ökologischen Fußabdrucks der Menschheit hervor, das die Anforderungen der Menschheit an die Erde ins Verhältnis zu ihrer Kapazität, diese zu erfüllen, setzt. Dabei werden jetzt verschiedene Formen von Grenzen (z.B. nach Gegenstandsfeldern oder Zeitspannen) sowie von Grenzüberschreitungen (z.B. Grenzüberschreitung mit Schwingungsvorgang und Grenzüberschreitung mit Zusammenbruch) differenziert. Ins Blickfeld treten jetzt auch stärker die Komplexität der Zusammenhänge (feedbacks, nichtlineare Beziehungen etc.) zwischen verschiedenen Formen von Grenzüberschreitung sowie die epistemischen Schwierigkeiten ihrer Erfassung. „Die Signale, die der wachsenden Bevölkerung und Wirtschaft die Grenzen aufzeigen, sind verzerrt, verschwommen und verwirrend, kommen mit Verzögerung an oder werden ignoriert. Daher erfolgen die Reaktionen auf diese Signale ebenfalls verzögert." (Meadows et al. 2006, S. 182) Von großer Bedeutung ist dabei die Figur der Kipppunkte oder auch tipping points. „Das Unheimliche daran ist, dass niemand weiß, wann ein tipping point erreicht ist, wie lange ein System belastbar ist, wo seine Bruchstellen liegen. All das zeigt sich erst im 
Kollaps. Ex post wird man gewusst haben, dass eine Grenze erreicht wurde, die man nicht zur Kenntnis genommen hat.“ (Horn 2014, S. 378, Herv. i. O.) Konzepte wie Grenzüberschreitung mit und ohne Zusammenbruch führen zu einer Verräumlichung und Verzeitlichung des ökologischen Grenzdenkens, das mit verschiedenen Formen von Überlastung rechnet und diese Überlastungen nicht als einmalige Brüche oder punktuelle Katastrophen, sondern als gedehnte, längerfristige Prozesse fasst. Populäre Bilder, die den vermeinten Zustand einer globalen Grenzüberschreitung erfassen, sind die des Fiebers, der Verschuldung oder das der Kontraproduktivität; eine alltagspolitische Übersetzung quantifizierter Grenzüberschreitungen ist etwa der Erdüberlastungstag (oder auch Welterschöpfungstag, Weltüberlastungstag, Ressourcenerschöpfungstag, Ökoschuldentag; im Englischen spricht man vom Earth Overshoot Day oder auch Ecological Debt Day), der den Tag eines Jahres bezeichnet, an dem die Menschheit beginnt, die hypothetisch angenommenen jährlich zur Verfügung stehenden natürlichen Ressourcen überzubeanspruchen. Ein sprachliches Charakteristikum der ,Epoche der Grenzüberschreitung' ist die wachsende Bedeutung der Vorsilbe über-, wie sie in Begriffen wie Übervölkerung, Übernutzung, Überfischung, Überweidung, Überentwicklung, Übersäuerung erscheint. Das Deutsche Wörterbuch erfasst unter dem Lemma "Grenze“ solcherart Konstruktionen in einer besonderen Gruppe und kommentiert, dass die Grenze hier das Gebiet des Billigen, Erlaubten, Rechtmäßigen, Gehörigen oder Schicklichen abschließt (vgl. Hübner 1919/1935, Sp. 143). Diese Einschätzung erscheint vor dem Hintergrund des aktuellen Sprachgebrauchs nachgerade harmlos und steht in einem deutlichen Kontrast zur existenziellen Härte der ökologischen Begriffe, wo die Grenzüberschreitung eher in die Zone der Gefahr, des Risikos, des Zusammenbruchs oder der Überlebensbedrohung hineinführt.

Zeitlich parallel zur Herausbildung des ökologischen Grenzforschungsparadigmas entsteht seit den 1980er Jahren ein Diskurs zur sogenannten Globalisierung, der gerade den zunehmenden Bedeutungsverlust von Grenzen behauptet, wobei hier vor allem die politisch-territorialen Grenzen des traditionellen Nationalstaates gemeint sind. Leitbegriffe und Topoi des Globalisierungsdiskurses sind etwa Entgrenzung, Verflüssigung, grenzenlose Welt oder vielfältige Verbindungen mit dem Adjektiv grenzüberschreitend (Handel, Verkehr, Mobilität, Austausch, Informationen, Prozesse etc.). In Bezug auf den ökologischen Grenzdiskurs heißt das, dass die Umweltprobleme nur im Rahmen internationaler Vereinbarungen und Kooperationen angegangen werden können. Zu den drastischen Folgen des ökologischen Grenzüberzugs gehört die schon seit einiger Zeit beobachtbare beschleunigte Verschiebung, natürlicher' Grenzen (z.B. der Verbreitungsgebiete von Tieren und Pflanzen, der Klimazonen, der Gletscher und Eisgebiete bis hin zur Veränderung des Verhältnisses von Land- und Wassermassen) sowie die Zunahme von Massenmigration, die schon heute zur Veränderung staatlicher Grenzregime führen. Im Spannungsfeld zwischen kapitalistischer Entgrenzung und (öko-)politischer Begrenzung stoßen gegensätzliche Grenzbegriffe aufeinander, die der Ausdruck unbewältigter Probleme und realer politischer Konflikte sind (vgl. Altvater/Mahnkopf 1996). Eine neue Kategorie, die in diesem Spannungsfeld entstanden ist, ist die des Klimaflüchtlings, die an Bedeutung sicher zunehmen wird. Die vielen neuen Grenzstreitigkeiten zeigen jedenfalls, dass die mit dem Diskurs zur Globalisierung verbundene Auffassung von der Antiquiertheit der Grenze (Anders 1979) voreilig bzw. irreführend war. 


\section{Fazit und Ausblick}

Die neuere Institutionalisierung der Grenzforschung in den border(land) studies und der Boundary-Forschung seit den 1990er-Jahren indiziert das Bewusstsein, dass sich Grenzen keineswegs ganz aufgelöst haben, sondern verschoben, verändert, diversifiziert und neu organisiert wurden, was sich in einer erstaunlichen Fülle neuer Qualifizierungen wie etwa unsichtbare, virtuelle, intelligente, elektronische, smarte oder mobile Grenzen ausdrückt. Diese vielfältigen Adjektivierungen verweisen auf neue Technologien und Praktiken der Grenzziehung und auf den Prozess einer Medialisierung und Diversifizierung von Grenzen, der zu einer stärkeren Verklammerung politisch-geografischer und kultureller Grenzbestimmungen führt, weil sich damit die Grenzen immer mehr in die Gesellschaften hineinverlagern. Wie der vorliegende Aufsatz anhand begriffshistorischer Untersuchungen gezeigt hat, stehen diese Prozesse in einer längeren Tradition, die mit der Entstehung moderner Staaten beginnt. Markant seit der Aufklärung dient der Grenzbegriff dann auch der theoretischen Reflexion spezifischer Merkmale der modernen Gesellschaft. Die ihr inhärente Dynamik führt nicht nur zur Temporalisierung des Grenzbegriffs, sondern darüber hinaus auch dazu, dass sich in ihrem Rahmen der Begriff zu einer spezifischen Epochensignatur entwickelt. Die Moderne als eine Ordnung, die „sich nur (noch) dynamisch zu stabilisieren vermag“ (Rosa 2016, S. 673, Herv. i. O.), erscheint als „Zeitalter der Grenzüberwindung“ bzw. „Grenzüberschreitung“. Die für sie charakteristische Dialektik von (ökonomischer, technologischer) Entgrenzung und (politischer, moralischer, ethischer) Begrenzung setzt als Widerspruchsbewegung immer neue Grenzbegriffe frei. Die Flexibilisierung und Entgrenzung des Grenzbegriffs führt so weit, dass sich der Begriff in der Gestalt des frontier-Begriffs als Expansionsgrenze selbst noch sein Gegenteil einzuverleiben scheint. Der systemisch eingebaute Steigerungsimperativ und die daraus resultierende Beschleunigungsdynamik werden mit Sicherheit zu einem weiteren Bedeutungszuwachs von Grenzkategorien führen, da die gesellschaftlichen Strukturen und Lebensverhältnisse im Zeichen von Globalisierung und Klimakatastrophe unter einen weiter steigenden Veränderungsdruck geraten. Die Verflüssigung verschiedener Grenzen und Grenzsysteme wird zu massiven Grenzstreitigkeiten und Grenzkonflikten und zur Entstehung neuer Grenzziehungspraktiken (Formen der Einund Ausschließung) führen. Die neuere Grenzforschung reagiert auf diese Entwicklungen mit dem Plädoyer für eine „erweiterte Grenzperspektive“ und eine interdisziplinäre Zusammenführung verschiedener Grenzforschungen und Grenzbegriffe, um der Komplexität und dem Zusammenspiel verschiedener Grenzregime sowie den Widersprüchen und Ungleichzeitigkeiten feldspezifischer Grenzbegriffe und Grenzpraktiken Rechnung zu tragen (vgl. Newman 2006; Gerst et al. 2018). Sie trägt damit in einer unsicherer und unübersichtlicher werdenden Welt zur Politisierung der (kultur-)wissenschaftlichen Forschungspraxis bei.

\section{Weiterführende Literatur}

Böckler, Stefan (2003): Grenze: Allerweltswort oder Grundbegriffe der Moderne? In: Archiv für Begriffsgeschichte. Bd. 45, Hamburg: Meiner, S. 167-220.

Febvre, Lucien (1928/1988): „Frontière“ - Wort und Bedeutung. In: Ders.: Das Gewissen des Historikers. Berlin: Wagenbach, S. 27-37.

Hübner, Arthur (1919/1935): [Art.] Grenze. In: Grimm, Jacob/Grimm, Wilhelm (Hrsg.): Deutsches Wörterbuch. Bd. 9, Leipzig: S. Hirzel, Sp. 124-148.

Medick, Hans (1995): Grenzziehungen und die Herstellung des politisch-sozialen Raums. Zur Begriffsgeschichte und politischen Sozialgeschichte der Grenzen in der frühen Neuzeit. In: Faber, Richard/Naumann, Barbara (Hrsg.): Literatur der Grenze - Theorie der Grenze. Würzburg: Königshausen \& Neumann, S. 211-224. 
Scattola, Merio (1997): Die Grenze der Neuzeit. Ihr Begriff in der juristischen und politischen Literatur der Antike und Frühmoderne. In: Rahn, Thomas/Bauer, Markus (Hrsg.): Die Grenze: Begriff und Inszenierung. Berlin: De Gruyter, S. 37-69.

\section{Literaturverzeichnis}

Altvater, Elmar/Mahnkopf, Birgit (1996): Grenzen der Globalisierung. Ökonomie, Ökologie und Politik in der Weltgesellschaft. Münster: Westfälisches Dampfboot.

Anders, Günther (1979): Die Antiquiertheit der Grenze. In: Ders.: Die Antiquiertheit des Menschen 2. Über die Zerstörung des Lebens im Zeitalter der dritten industriellen Revolution. München: Beck, S. 208-209.

Anders, Günther (1956/2002): Die Antiquiertheit des Menschen 1. Über die Seele im Zeitalter der zweiten industriellen Revolution. München: Beck.

Arndt, Ernst Moritz (1813/1845): Der Rhein, Teutschlands Strom, aber nicht Teutschlands Gränze. In: Ders.: Schriften für und an seine lieben Deutschen, 2. Theil. Leipzig: Weidmannsche Buchhandlung, S. 1-66.

Barth, Reinhard (2018): Die Vermessung der Erde. Die Geschichte der Kartografie von der Papyrusrolle bis zum GPS. München: Delphin.

Bettelheim, Bruno (1943/1982): Die äußerste Grenze. In: Ders.: Erziehung zum Überleben. Zur Psychologie der Extremsituation. München: DTV, S. 11-27.

Blumenberg, Hans (1987): Die Sorge geht über den Fluß. Frankfurt/M.: Suhrkamp.

Böckler, Stefan (2003): Grenze: Allerweltswort oder Grundbegriffe der Moderne? In: Archiv für Begriffsgeschichte. Hamburg: Meiner, Bd. 45, S. 167-220.

Böckler, Stefan (2007): ,Grenze` und frontier: Zur Begriffs- und Sozialgeschichte zweier Schließungsparadigmen der Moderne. In: Deger, Petra/Hettlage, Robert (Hrsg.): Der europäische Raum. Die Konstruktion europäischer Grenzen. Wiesbaden: Springer, S. 25-48.

Bredow, Wilfried von (2014): Grenzen. Eine Geschichte des Zusammenlebens vom Limes bis Schengen. Berlin: Theiss.

Descartes, René (1626-28/1972): Regeln zur Ausrichtung der Erkenntniskraft. Krit. rev., übers. und hrsg. v. Heinrich Springmeyer et al., Hamburg: Meiner.

Dix, Arthur (1922a): Politische Erdkunde. Breslau: Verlag Ferdinand Hirt.

Dix, Arthur (1922b): Politische Geographie. Weltpolitisches Handbuch. München/Berlin: R. Oldenbourg.

Du Bois-Reymond, Emil (1873): Über die Grenzen des Naturerkennens. Ein Vortrag. Leipzig: Veit \& Comp.

Eppler, Erhard (1975): Ende oder Wende. Von der Machbarkeit des Notwendigen. München: DTV.

Ersch, Johann Samuel/Gruber, Johann Gottfried (1818-1889): [Art.] Grenze. In: Allgemeine Enzyklopädie der Wissenschaften und Künste, Erste Section A-G, 90. Teil. Leipzig: Brockhaus, S. 185-234.

Febvre, Lucien (1928/1988): „Frontière“ - Wort und Bedeutung. In: Ders.: Das Gewissen des Historikers. Berlin: Wagenbach, S. 27-37.

Fichte, Johann Gottlieb (1808/1846): Reden an die deutsche Nation. In: Ders.: Sämmtliche Schriften, hrsg. v. Immanuel Hermann Fichte, Bd. 7. Berlin: Veit \& Comp., S. 258-499.

Flink, Tim/Kaldewey, David (2018): The Language of Science Policy in the Twenty-First Century: What comes after Basic and Applied Research? In: Kaldewey, David/Schauz, Désirée (Hrsg.): Basic and Applied Research. The Language of Science Policy in the Twentieth Century. New York/ Oxford: Berghahn, S. 251-284.

Fulda, Friedrich (1974): [Art.] Grenze, Schranke. In: Ritter, Joachim (Hrsg.): Historisches Wörterbuch der Philosophie, Bd. 3. Darmstadt: Wissenschaftliche Buchgesellschaft, S. 875-877.

Gerst, Dominik/Klessmann, Maria/Krämer, Hannes/Sienknecht, Mitja/Ulrich, Peter (2018): Komplexe Grenzen. Aktuelle Perspektiven der Grenzforschung. In: Berliner Debatte Initial 29, H. 1, S. 3-11.

Gervinus, Georg Gottfried (1842): Neuere Geschichte der poetischen National-Literatur der Deutschen. Leipzig: Wilhelm Engelmann.

Grossmann, Henryk (1929): Das Akkumulations- und Zusammenbruchsgesetz des kapitalistischen Systems (zugleich eine Krisentheorie). Leipzig: Hirschfeld.

Grossmann, James R. (1994): The frontier in American culture. Berkeley/Los Angeles/London: University of California Press.

Gruhl, Herbert (1975): Ein Planet wird geplündert. Die Schreckensbilanz unserer Politik. Frankfurt/M.: Fischer.

Haug, Wolfgang Fritz (2001): [Art.] Grenzen der Dialektik. In: Haug, Wolfgang Fritz (Hrsg.): Historischkritisches Wörterbuch des Marxismus. Bd. 5, Hamburg: Argument, S. 957-962. 
Haushofer, Karl (1927): Grenzen in ihrer geographischen und politischen Bedeutung. Berlin-Grunewald: Kurt Vowinckel.

Hegel, Georg Wilhelm Friedrich (1830/1970): Enzyklopädie der philosophischen Wissenschaften im Grundrisse, Erster Teil: Die Wissenschaft der Logik. Mit den mündlichen Zusätzen, neu editierte Ausgabe. In: Ders.: Werke in 20 Bänden, Bd. 8. Frankfurt/M.: Suhrkamp.

Hegel, Georg Wilhelm Friedrich (1812-16/1969): Wissenschaft der Logik. In: Ders., Werke in 20 Bänden, Bd. 5. Frankfurt/M.: Suhrkamp.

Helmholt, Hans Ferdinand (1896): Die Entwickelung der Grenzlinie aus dem Grenzsaume im alten Deutschland. In: Historisches Jahrbuch, 17. Jg., S. 235-264.

Holdich, Thomas H. (1916): Geographical problems in Boundary making. In: The Geographical Journal 47, H. 6, S. 421-440.

Hoke, R. (1971): [Art.] Grenze. In: Erler Adalbert/Kaufmann, Ekkehard (Hrsg.): Handwörterbuch zur deutschen Rechtsgeschichte. Berlin: Erich Schmidt, S. 1801-1806.

Horn, Eva (2014): Zukunft als Katastrophe. Frankfurt/M.: Fischer.

Hübner, Arthur (1919/1935): [Art.] Grenze. In: Grimm, Jacob/Grimm, Wilhelm (Hrsg.): Deutsches Wörterbuch, Bd. 9. Leipzig: S. Hirzel, Sp. 124-148.

Illich, Ivan (1975): Selbstbegrenzung. Reinbek bei Hamburg: Rowohlt.

Jaspers, Karl (1931/1964): Die geistige Situation der Zeit. Berlin: De Gruyter.

Jaspers, Karl (1932): Existenzerhellung. Philosophie II. Berlin: Springer.

Jünger, Ernst (1932/1982): Der Arbeiter. Herrschaft und Gestalt. Stuttgart: Klett-Cotta.

Kant, Immanuel (1766/1912): Träume eines Geistersehers. In: Ders.: Gesammelte Schriften, hg. v. d. Königl. Preußischen Akademie der Wissenschaften, Bd. II. Berlin: Reimer.

Keynes, John Maynard (1936/1983): Allgemeine Theorie der Beschäftigung, des Zinses und des Geldes. Berlin: Duncker \& Humblot.

Kolb, Herbert (1989): Zur Frühgeschichte des Wortes ,Grenze'. In: Heitmann, Klaus/Kolb, Herbert/Mehl Dieter (Hrsg.): Archiv für das Studium der neueren Sprachen und Literaturen, Bd. 226, 141. Jg. Berlin: Erich Schmidt, S. 344-356.

Kurz, Anton (1821): Versuch einer Entwicklung der Grundsätze, nach welche die Zweckmäßigkeit des Staatsorganismus in konstitutionellen Monarchien zu beurtheilen ist. München: Lentner.

La Pradelle, Paul de (1928): La frontiere. Etude de droit International. Paris: Les Éditions Internationales.

Luxemburg, Rosa (1913/1975): Die Akkumulation des Kapitals. Ein Beitrag zur Erklärung des Imperialismus. Berlin: Dietz.

Mansholt, Sicco (1974): Die Krise: Europa und die Grenzen des Wachstums. Reinbek bei Hamburg: Rowohlt.

Marx, Karl (1857-58/1974): Grundrisse der Kritik der Politischen Ökonomie. Berlin: Dietz.

Matznetter, Josef (1977): Einleitung. In: Ders. (Hrsg.): Politische Geographie. Darmstadt: Wissenschaftliche Buchgesellschaft, S. 1-27.

Maull, Otto (1919): Geographische Staatsstruktur und Staatsgrenzen. In: Kartographische Zeitschrift 8, S. 129-136.

Maull, Otto (1925): Politische Geographie. Berlin: Gebr. Bornträger.

Meadows, Donella/Randers, Jørgen/Meadows, Dennis (1973): Die Grenzen des Wachstums. Bericht des Club of Rome zur Lage der Menschheit. Reinbek bei Hamburg: Rowohlt.

Meadows, Donella/Randers, Jørgen/Meadows, Dennis (1993): Die neuen Grenzen des Wachstums. Reinbek bei Hamburg: Rowohlt.

Meadows, Donella/Randers, Jørgen/Meadows, Dennis (2006): Grenzen des Wachstums. Das 30-Jahre-Update. Signal zum Kurswechsel. Stuttgart: Hirzel.

Medick, Hans (1995): Grenzziehungen und die Herstellung des politisch-sozialen Raums. Zur Begriffsgeschichte und politischen Sozialgeschichte der Grenzen in der frühen Neuzeit. In: Faber, Richard/Naumann, Barbara (Hrsg.): Literatur der Grenze - Theorie der Grenze. Würzburg: Königshausen \& Neumann, S. 211-224.

Mesarović, Mihajlo/Pestel, Eduard (1974): Menschheit am Wendepunkt. 2. Bericht des Club of Rome zur Weltlage. Stuttgart: DVA.

Minghi, Julian V. (1977): Grenzen in der Politischen Geographie. In: Matznetter, Josef (Hrsg.): Politische Geographie. Darmstadt: Wissenschaftliche Buchgesellschaft, S. 338-389.

Newman, David (2006): Borders and Bordering, Towards an Interdisciplinary Dialogue. In: European Journal of Social Theory 9, H. 2, S. 171-186.

Peccei, Aurelio/Siebker, Manfred (1974): Die Grenzen des Wachstums - Fazit und Folgestudien. Reinbek bei Hamburg: Rowohlt.

Penck, Albrecht (1916): Die österreichische Alpengrenze. Stuttgart: Engelhorn. 
Penck, Albrecht (1917): Über politische Grenzen. Rede zum Antritt des Rektorates der Königlichen Friedrich-Wilhelms-Universität in Berlin. Berlin: Norddeutsche Druckerei und Verlagsanstalt.

Petersen, Hans (1996): Der Terminus Frontier im Amerikanischen. Ursprung, Wandlung und Wirkung einer Sprach- und Denkform. Kassel: Bräuning und Rudert.

Plessner, Helmuth (1924/2002): Grenzen der Gemeinschaft. Eine Kritik des sozialen Radikalismus. Frankfurt/M.: Suhrkamp.

Plessner, Helmuth (1967/2003): Das Problem der Unmenschlichkeit. In: Ders.: Gesammelte Schriften, Bd. VIII. Conditio Humana. Frankfurt/M.: Suhrkamp, S. 328-337.

Ratzel, Friedrich (1892): Über allgemeine Eigenschaften der geographischen Grenzen und über die politische Grenze (Bericht über die Verhandlungen der königlich sächsischen Gesellschaft der Wissenschaften zu Leipzig). Leipzig: Hirzel, S. 53-104.

Ratzel, Friedrich (1901): Der Lebensraum. Eine biogeographische Studie, unveränderter Nachdruck aus: Festgabe für Albert Schäffle. Tübingen: Laupp, S. 101-189.

Ratzel, Friedrich (1896/1977): Die Gesetze des räumlichen Wachstums der Staaten. Ein Beitrag zur wissenschaftlichen politischen Geographie. In: Matznetter, Josef (Hrsg.): Politische Geographie. Darmstadt: Wissenschaftliche Buchgesellschaft, S. 29-53.

Rickert, Heinrich (1896/1902): Grenzen der naturwissenschaftlichen Begriffsbildung. Tübingen/Leipzig: Mohr.

Rodbertus-Jagetzow, Carl v. (1869): Zur Erklärung und Abhülfe der heutigen Credithnoth des Grundbesitzes. Bd. 2: Zur Abhülfe. Berlin: H. Bahr.

Rosa, Hartmut (2016): Resonanz. Eine Soziologie der Weltbeziehung. Berlin: Suhrkamp.

Saner, Hans (1974): [Art.] Grenzsituation. In: Ritter, Joachim (Hrsg.): Historisches Wörterbuch der Philosophie, Bd. 3. Darmstadt: Wissenschaftliche Buchgesellschaft, S. 877-878.

Scattola, Merio (1997): Die Grenze der Neuzeit. Ihr Begriff in der juristischen und politischen Literatur der Antike und Frühmoderne. In: Rahn, Thomas/Bauer, Markus (Hrsg.): Die Grenze: Begriff und Inszenierung. Berlin: De Gruyter, S. 37-69.

Schneider, Ute (2006): Die Macht der Karten. Eine Geschichte der Kartographie vom Mittelalter bis heute. Darmstadt: Primus.

Schöne, Emil (1911): Politische Geographie. Leipzig: Teubner.

Schmale, Wolfgang (1989): ,Grenze' in der deutschen und französischen Frühneuzeit. In: Schmale, Wolfgang/Stauber, Reinhard (Hrsg.): Menschen und Grenzen in der frühen Neuzeit. Berlin: Berlin Verlag, S. 50-75.

Schumpeter, Joseph (1918): Zur Soziologie der Imperialisten. In: Archiv für Sozialwissenschaft und Sozialpolitik, Bd. 46, S. 1-39 und 275-310.

Sieger, Robert (1917/18): Zur politisch-geographischen Terminologie. Natürliche und politische Grenzen. In: Zeitschrift der Gesellschaft für Erdkunde zu Berlin, S. 504-529 (1917) und 48-70 (1918).

Sieger, Robert (1921): Grenzen. In: Deutsche Arbeit, 21. Jg., H. 3, Reichenberg-Berlin: o.V.

Sieger, Robert (1926): Die geographische Lehre von den Grenzen und ihre praktische Bedeutung. In: Verhandlungen des 21. Deutschen Geographentags. Breslau: Hirt, S. 197-211.

Simmel, Georg (1908/1992): Soziologie. Untersuchungen über die Formen der Vergesellschaftung. In: Ders.: Gesamtausgabe, hrsg. v. Otthein Rammstedt, Bd. 11. Frankfurt/M.: Suhrkamp.

Simmel, Georg (1918/1999): Lebensanschauung. In: Ders., Gesamtausgabe, hrsg. v. Otthein Rammstedt, Bd. 16. Frankfurt/M.: Suhrkamp, S. 209-425.

Seweloh, Carl (1808): Grenzrevision und Grenzregulierung in rechtlicher und mathematischer Hinsicht. Fulda.

Sölch, Johann (1924a): Die Auffassung der ,natürlichen Grenzen` in der wissenschaftlichen Geographie. Innsbruck.

Sölch, Johann (1924b): Die Brennergrenze eine ,natürliche‘ Grenze? In: Tiroler Heimat: Jahrbuch für Geschichte und Volkskunde, Bd. 5/6, Innsbruck: Tyrolia, S. 58-96.

Spengler, Oswald (1931): Der Mensch und die Technik. Berlin: Voltmedia.

Steffens, Henrik (1808-09/1956): Vorlesungen über die Idee der Universitäten. In: Anrich, Ernst (Hrsg.): Die Idee der deutschen Universität. Die fünf Grundschriften aus der Zeit ihrer Neubegründung durch klassischen Idealismus und romantischen Realismus. Darmstadt: Gentner.

Supan, Alexander (1918): Leitlinien der allgemeinen politischen Geographie. Leipzig: Veit \& Comp.

Turner, Frederick Jackson (1893/1920): The Significance of the Frontier in American History. In: Ders.: The Frontier in American History. New York: Henry Holt \& Co, S. 1-38.

Turner, Frederick Jackson (1914): The Frontier in American History. New York: Henry Holt \& Co.

Vogel, Walter (1926): Zur Lehre von den Grenzen und Räumen. In: Geographische Zeitschrift, S. 191198. 
Wieland, Martin (1781/1928): Über den Hang der Menschen, an die Magie und Geisthererscheinungen zu glauben. In: Ders.: Gesammelte Schriften, hrsg. v. Wilhelm Kurrelmeyer, 1. Abteilung, Bd. IX (14). Berlin: Weidmannsche Buchhandlung, S. 322-331.

Wieland, Martin (1788/1930): Das Geheimniß des Kosmopoliten-Ordens. In: Ders., Gesammelte Schriften, hrsg. v. Wilhelm Kurrelmeyer, Bd. X (15). Berlin: Weidmannsche Buchhandlung, S. 207-229.

Wieser, Friedrich von (1884): Über den Ursprung und die Hauptgesetze des wirtschaftlichen Werthes. Wien: Hölder.

Wokart, Norbert (1995): Differenzierungen im Begriff ,Grenze‘. Zur Vielfalt eines scheinbar einfachen Begriffs. In: Faber, Richard/Naumann, Barbara (Hrsg.): Literatur der Grenze - Theorie der Grenze. Würzburg: Königshausen \& Neumann, S. 275-289.

Zedler, Johann Heinrich (1731-1754): [Art.] Grentzen. In: Ders.: Großes vollständiges Universal-Lexicon aller Wissenschafften und Künste, Bd. 11. Leipzig/Halle: Zedler, S. 831-843. 